Cerebrovasc Dis 2008;26:447-448

DOI: $10.1159 / 000157630$

\section{Normalization of Brain Tissue Lactate after Hyperbaric Oxygen Therapy in a Progressive Stroke Patient}

John-Ih Lee a , Hans-Joerg Wittsack ${ }^{\mathrm{b}}$, Andreas Christaras c, Falk Roland Miese ${ }^{\mathrm{b}}$, Mario Siebler ${ }^{\mathrm{a}}$

${ }^{a}$ Department of Neurology, ${ }^{b}$ Institute of Diagnostic

Radiology and Neuroradiology, and 'Hyperbaric Oxygen Unit, Department of Trauma and Hand Surgery, Heinrich Heine University, Düsseldorf, Germany

\section{Introduction}

In contrast to experimental results $[1,2]$, clinical trials with hyperbaric oxygen therapy (HBOT) have failed to show benefit in acute ischemic stroke [3] and its application has been discussed controversially $[4,5]$. We used proton magnetic resonance spectroscopic imaging $\left({ }^{1} \mathrm{H}-\mathrm{MRS}\right)$ to detect the effect of HBOT in a progressive ischemic stroke patient.

Fig. 1. a Axial MR time-of-flight angiography indicates bilateral occlusion of the internal carotid arteries (3D gradient echo sequence, $\mathrm{TR}=22 \mathrm{~ms}, \mathrm{TE}=3.7 \mathrm{~ms}, \mathrm{FOV}=105 \times 200 \mathrm{~mm}$, image matrix $=202 \times 384,3$ slabs, 40 image sections, section thickness $0.65 \mathrm{~mm})$. b Axial diffusion weighted-image brain MRI shows new ischemia in the centrum semiovale of the right hemisphere (arrowheads) pointing to an acute hemodynamic watershed infarct (Echo planar imaging sequence, $\mathrm{TR}=3,200 \mathrm{~ms}, \mathrm{TE}=92 \mathrm{~ms}$, image matrix $=192 \times 192, \mathrm{FOV}=230 \mathrm{~mm}$, slice thickness $=$ $5 \mathrm{~mm}$, gap $=1.5 \mathrm{~mm}, 20$ slices, $\mathrm{b}$-value $=0 ; 500 ; 1,000 \mathrm{~s} / \mathrm{mm}^{2}$ in 3 orthogonal directions combined to a diffusion-weighted imaging trace image). c Axial $\mathrm{T}_{2}$-weighted sequence shows voxel for ${ }^{1} \mathrm{H}$ MRS planning prior to HBOT (Turbo spin echo sequence, $\mathrm{TR}=$ $5,000 \mathrm{~ms}, \mathrm{TE}=100 \mathrm{~ms}$, section thickness $=5 \mathrm{~mm}$, gap $1.5 \mathrm{~mm}$, FOV $=240 \mathrm{~mm}$, image matrix $=384 \times 512,2$ averages, images acquired in 3 orthogonal directions). d Corresponding ${ }^{1} \mathrm{H}$-MRS reveals a negative double peak at 1.3 parts per million (ppm) attributable to lactate in the new ischemic area prior to HBOT. Other peaks represent $\mathrm{N}$-acetyl-aspartate (NAA), choline (Cho) and creatine (Cr, Cr2) (Spectroscopic imaging sequence, $\mathrm{TR}=1,700$ $\mathrm{ms}, \mathrm{TE}=135 \mathrm{~ms}, \mathrm{FOV}=160 \mathrm{~mm}$, image matrix $=16 \times 16, \mathrm{sec}-$ tion thickness $=15 \mathrm{~mm}$, voxel size $=10 \times 10 \times 15 \mathrm{~mm}, 3$ aver ages). e Axial $\mathrm{T}_{2}$-weighted sequence shows voxel for ${ }^{1} \mathrm{H}$-MRS planning after 8 HBOT units. f After 8 HBOT units corresponding ${ }^{1} \mathrm{H}$-MRS detects no pathologic lactate peak in the same area.
Case Report

A 56-year-old right-handed woman was admitted because of worsening of a right-sided hemiparesis and dysphasia for 20 days. Vascular risk factors were smoking (40 pack-years) and a family history of cerebrovascular diseases. Neurological examination showed decreased spontaneous movement, expressive dysphasia, a right-sided hemiparesis and a left-sided arm paresis (MRC scale: $3+/ 5$ ). Duplex sonography (Aplio XV; Toshiba), magnetic resonance angiography (fig. 1a; 3 Tesla MRT; Trio; Siemens) and cere-
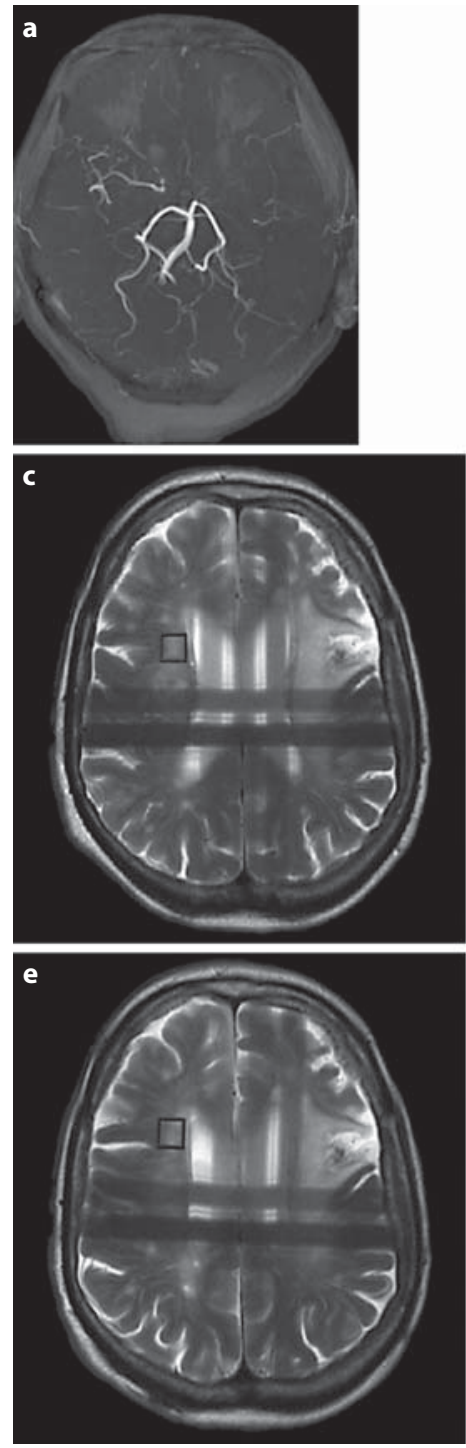
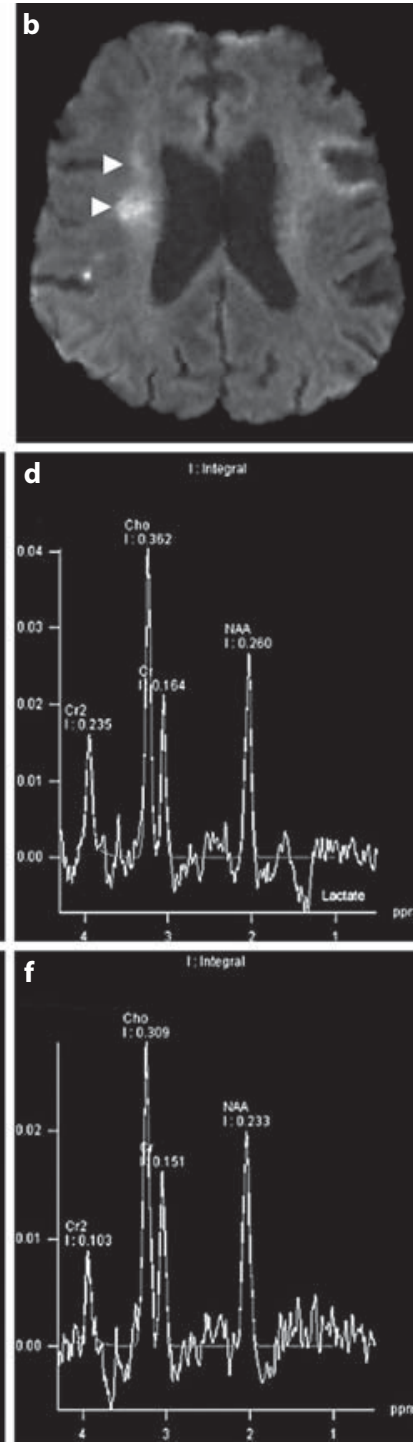

\section{KARGER}

Fax +41613061234 E-Mail karger@karger.ch www.karger.com
(C) 2008 S. Karger AG, Basel

$1015-9770 / 08 / 0264-0447 \$ 24.50 / 0$ 
bral digital subtraction angiography revealed a bilateral occlusion of the internal carotid arteries and the right vertebral artery, with the retrograde right ophthalmic artery as the only vessel supplying the brain. Further MRI sequences showed, besides old left hemispheric infarcts, new ischemic lesions in the right centrum semiovale, pointing to a recent watershed infarct (fig. 1b). ${ }^{1} \mathrm{H}$ MRS revealed a lactate peak in these ischemic areas (fig. 1c, d). A $550 \mathrm{MBq}{ }^{99 \mathrm{~m} T c-H M P A O}$ brain perfusion scintigraphy showed hypoperfusion in both middle and anterior cerebral artery territories with severely impaired functional cerebrovascular reserve capacity.

Because of the hemodynamic cause of these infarcts, early treatment consisted of antiplatelet agents and hypotension prevention. We decided to support the oxygen supply of the brain with HBOT in order to bridge the time until neurosurgical treatment with an extracranial to intracranial bypass.

Hyperbaric oxygen treatment units were administered once daily for 8 days. Each treatment unit consisted of 90 min surplus pressure with $100 \%$ oxygen at 2.0 bar in a hyperbaric chamber (Sayers-Hebold, Germany). Pressure and duration of each HBOT session were chosen according to human and experimental animal data in cerebral ischemia $[1-3,6]$.

After 8 hyperbaric oxygen treatment units and 11 days, an MRI and ${ }^{1} \mathrm{H}$-MRS detected neither new ischemic lesions nor lactate peaks in the same areas (fig. le, f) and the patient showed clinical improvement. The patient was then treated with an extracranial to intracranial bypass, which preserved the spectroscopic and clinical result.

\section{Discussion}

There is no consensus on the efficacy of HBOT in ischemic stroke $[4,5]$. Here, the first MRI showed new ischemic lesions and, additionally, ${ }^{1} \mathrm{H}$-MRS indicated hypoxic tissue with lactate due to severely impaired cerebral perfusion.

The absence of the lactate peak in the new ischemic areas after HBOT might be induced by HBOT rather than by spontaneous decline, since lactate has been reported to remain at constantly high levels for more than 1 month after ischemia [7] and the period between the first and second ${ }^{1} \mathrm{H}-\mathrm{MRS}$ was only 11 days.

Furthermore, lactate has been described as a prognostic marker for a significantly poorer clinical outcome in ischemic stroke [8]. The number of HBOT sessions was set at 8, based on the time until neurosurgical treatment could be performed.

Our results suggest that HBOT increases brain tissue oxygenation in ischemic areas and may serve as a treatment option in severely hemodynamically compromised patients until definitive revascularization.

${ }^{1} \mathrm{H}-\mathrm{MRS}$ may be useful for monitoring HBOT efficacy, although more experimental and clinical data are necessary to confirm our assumption.

\section{References}

-1 Beynon C, Sun L, Marti HH, Heiland S, Veltkamp R: Delayed hyperbaric oxygenation is more effective than early prolonged normobaric hyperoxia in experimental focal cerebral ischemia. Neurosci Lett 2007; 425:141-145.

2 Henninger N, Küppers-Tiedt L, Sicard KM, Günther A, Schneider D, Schwab S: Neuroprotective effect of hyperbaric oxygen therapy monitored by MR-imaging after embolic stroke in rats. Exp Neurol 2006 201:316-323.
-3 Rusyniak DE, Kirk MA, May JD, Kao LW, Brizendine EJ, Welch JL, Cordell WH, Alonso RJ: Hyperbaric oxygen therapy in acute ischemic stroke: results of the Hyperbaric Oxygen in Acute Ischemic Stroke Trial Pilot Study. Stroke 2003;34:571-574.

4 Helms AK, Whelan HT, Torbey MT: Hyperbaric oxygen therapy of cerebral ischemia. Cerebrovasc Dis 2005;20:417-426.

5 Singhal AB: A review of oxygen therapy in ischemic stroke. Neurol Res 2007:29:173-183

6 Sarno JE, Rusk HA, Diller L, Sarno MT: The effect of hyperbaric oxygen on the mental and verbal ability of stroke patients. Stroke 1972;3:1015 .

-7 Houkin K, Kamada K, Kamiyama H, Iwasaki Y, Abe H, Kashiwaba T: Longitudinal changes in proton magnetic resonance spectroscopy in cerebral infarction. Stroke 1993;24:1316-1321.

${ }_{8}$ Federico F, Simone IL, Lucivero V, Giannini P, Laddomada G, Mezzapesa DM, Tortorella C: Prognostic value of proton magnetic resonance spectroscopy in ischemic stroke. Arch Neurol 1998;55:489494.

\section{Mario Siebler, MD}

Department of Neurology, Heinrich Heine University

Moorenstrasse 5

DE-40225 Düsseldorf (Germany)

Tel. +4921181 18464, Fax +492118118485

E-Mail siebler@uni-duesseldorf.de

\section{Cerebrovasc Dis 2008;26:448-449 \\ DOI: 10.1159/000157631}

\section{Moyamoya Syndrome Associated with Optic Nerve Coloboma and Mental Retardation}

\section{Konstantinos Spengos, Sofia Vassilopoulou,} Evangelos Anagnostou, Georgios Paraskevas, Vassilios P. Zis

Department of Neurology, University of Athens School of Medicine, Eginition University Hospital, Athens, Greece

Moyamoya is an intracranial arteriopathy of undetermined etiology that is characterized by the progressive obliteration of the major arteries of the anterior and, in some cases, the posterior cerebral circulation, and their replacement by a meshwork of small collateral vessels at the base of the brain. Moyamoya encompasses two distinct entities: (1) Moyamoya disease which is mostly seen among children and young adults with no other pathological conditions; (2) Moyamoya syndrome which is mostly seen in western countries, and its arterial abnormalities are considered as epiphenomena in response to another vascular process and have been associated with a series of conditions [1].

Mental retardation has been described in several patients with Down syndrome associated with Moyamoya [2]. In addition, ocular malformations, such as morning glory disk anomaly, optic disc and infrapapilar choroidal coloboma, have been reported in a small number of Moyamoya patients [3, 4]. 